\title{
Forging a sustainable future for astronomy
}

The climate crisis is no longer a prediction for the future, it is happening here and now. Astronomers have realized that they need to become part of the solution and are working towards reducing their own carbon footprint as well as communicating an astronomical perspective.

\section{Leonard Burtscher, Hannah Dalgleish, Didier Barret, Tobias Beuchert, Abhijeet Borkar, Faustine Cantalloube, Abigail Frost, Victoria Grinberg, Natasha Hurley-Walker, Violette Impellizzeri, Mathieu Isidro, Knud Jahnke and Michelle Willebrands}

T he climate crisis is real and humans are causing $i^{1}$ : the urgency of these scientific facts is becoming increasingly clear. Developing nations such as low-lying Bangladesh have experienced the impacts of climate change with devastating floods in recent years ${ }^{2}$, while richer nations in the global north suffer record wildfires ${ }^{3}$, heatwaves and floods. Indeed, a study ${ }^{4}$ conducted earlier this year by the Yale Program on Climate Change Communication found that internationally, a large majority of people have understood that everyone is vulnerable in a deteriorating climate, and encourages immediate and significant action. And yet, current climate action is too little and too slow to reduce emissions quickly enough to keep within $1.5^{\circ} \mathrm{C}$ of global heating, the goal of the 2015 Paris Agreement. More awareness, and more action, is essential. Fundamentally it is governments and corporations that must act, but actions by individuals and groups have power too, especially when they come from wealthy people whose contributions to greenhouse gas emissions are disproportionally large (Fig. 1).

The carbon footprint of astronomers is substantial, too. For instance, the average emissions associated with visiting a single in-person conference are similar to the annual per capita emissions of developing countries ${ }^{5}$. And yet astronomers can contribute to solving the climate crisis in two key ways. Firstly, by demonstrating how global collaboration is possible without burning fossil fuels, and secondly, through communicating the climate crisis from an astronomical perspective ${ }^{6-8}$.

Discussing, and reducing, our own emissions as astronomers is vital for a number of reasons. The most fundamental is the moral argument: we know that our emissions are causing harm, so reducing them is the ethical choice. But we also need to reduce emissions for our own discipline's sake $^{9}$ (Fig. 2). Countries signing up to net-zero targets necessitate the need to perform carbon-neutral research; the sooner

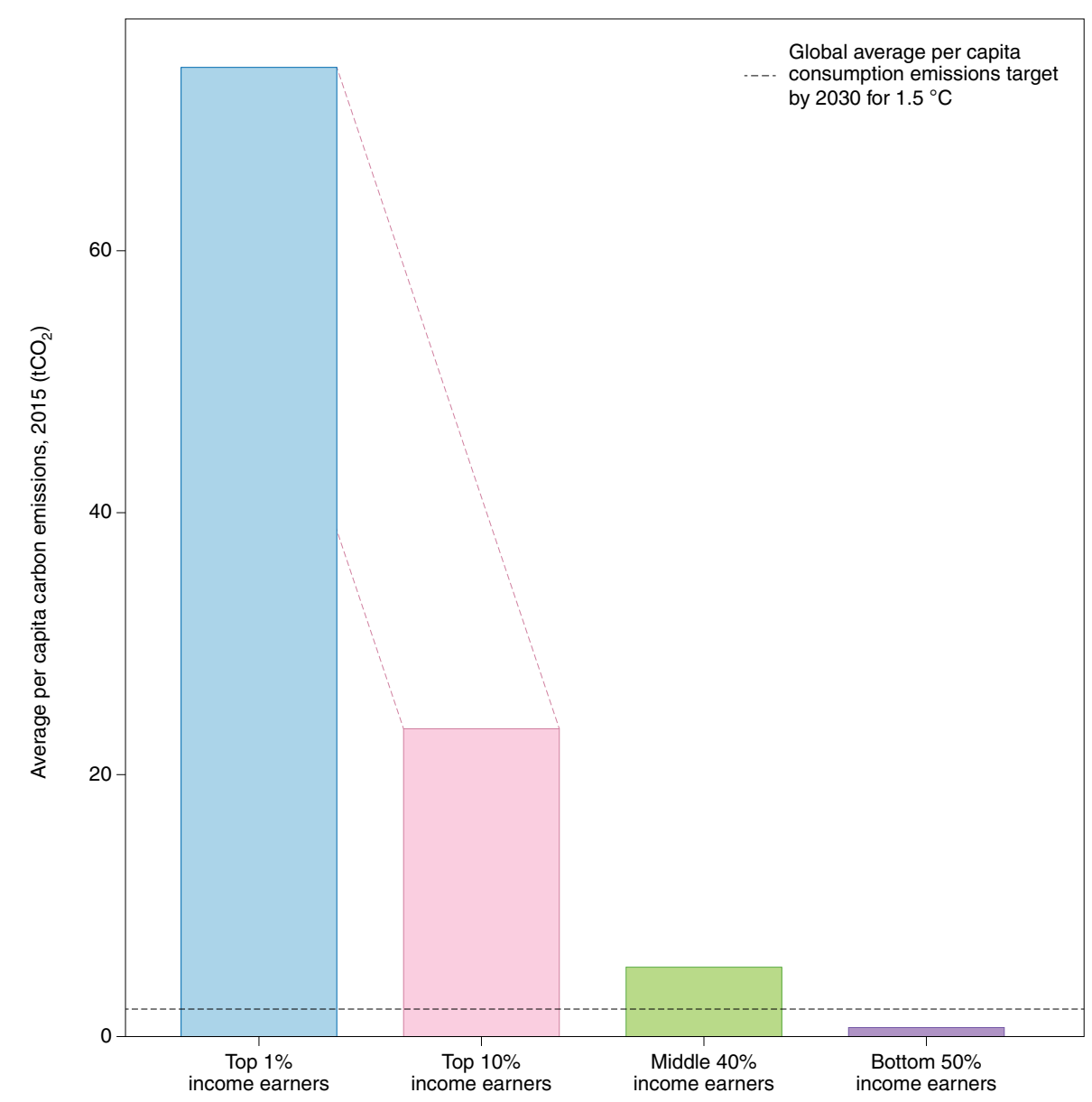

Fig. 1 | Who is responsible for carbon emissions world-wide? The richest 1\% (income >US $\$ 109,000$ ) of the population produce $15 \%$ of emissions and the $10 \%$ richest (>US $\$ 38,000$ ) produce $48 \%$ of emissions. This shows that our lifestyle has the highest impact on our planet; wealthy people therefore have the highest imperative to change behaviour. Data taken from ref. ${ }^{28}$.

we prepare, the better we will manage the transition for our own discipline. Finally, we need to reduce emissions in order to make ourselves credible actors in the much larger societal fight against the climate crisis. How can we convincingly tell the general public and policy makers that 'there is no planet B' while hopping onto the next intercontinental flight for a conference at a fancy location?
To support both climate action within astronomy and to help astronomers communicate the climate crisis, the grass-roots organization Astronomers for Planet Earth (A4E) was founded in 2019. In two years, A4E has rapidly grown to a global organization connecting more than 1,100 research astronomers, astronomy educators, and students ${ }^{7}$. A4E members 


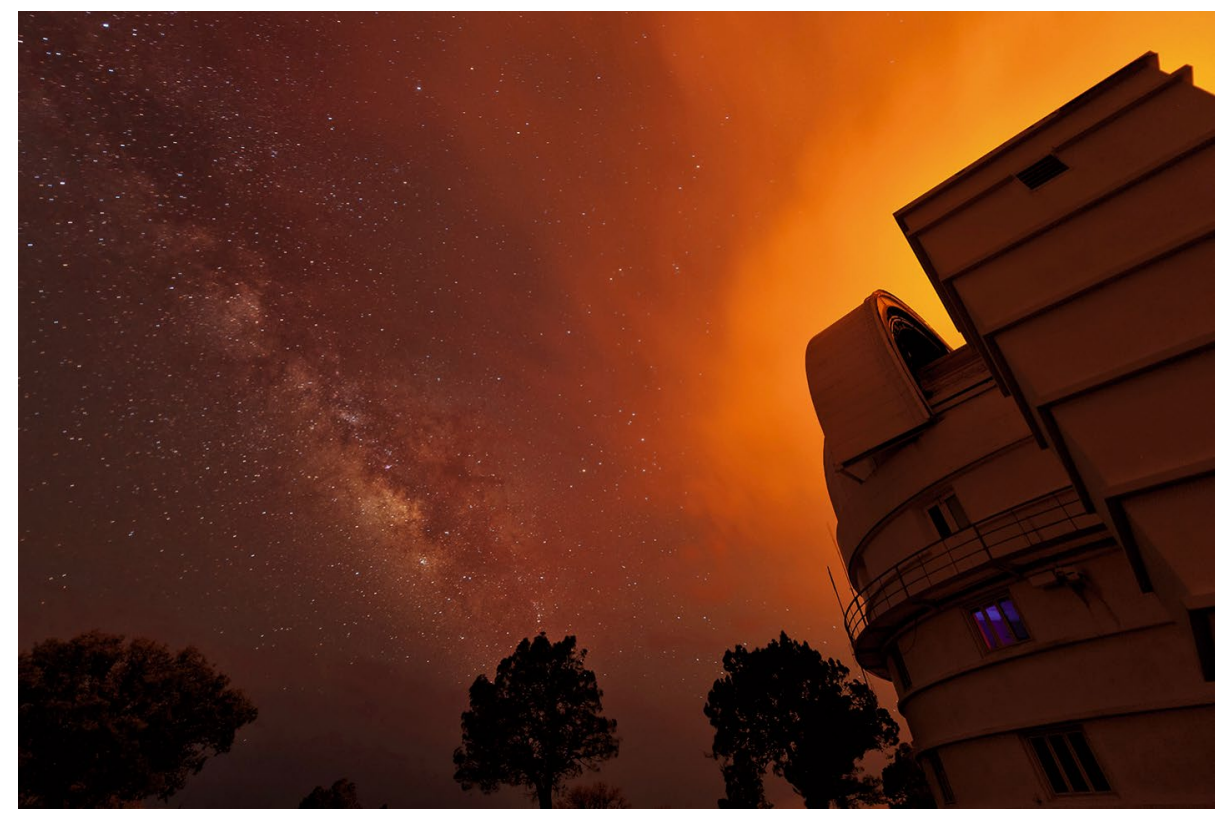

Fig. 2 | A wildfire endangers the McDonald Observatory in Fort Davis, Texas. Astronomers need to reduce their carbon footprint also for their own sake, since ground-based telescopes will become unreliable for research in an extreme climate. Credit: John Davis/Stocktrek Images/Getty

have organized several events, such as sessions and workshops at several major conferences including the annual meetings of the American and European astronomical societies as well as a special symposium in collaboration with the Astronomical Society of the Pacific; a webinar series that educates astronomers about the science of climate change; and numerous public lectures that share the astronomical perspective on our 'pale blue dot'. A4E has also run campaigns to call for climate action within astronomy and beyond, most notably the recent open letter to astronomy institutions that has been signed by about 3,000 astronomy professionals to date.

In the last year, a number of studies have been published analysing and quantifying in detail the carbon footprint of various entities of astronomical research: observatories ${ }^{10-13}$, institutes ${ }^{14,15}$ and conferences ${ }^{5}$. The main sources of $\mathrm{CO}_{2}$ are electricity (for operating telescopes, computing, data transport/ storing), flights (especially intercontinental), purchases (computers and other equipment), buildings (mostly heating and cooling), and materials for construction (mostly steel and concrete) (Fig. 3). The relative contribution of each varies both regionally (for example, due to differences in the carbon intensity of the local electricity generation) and per institute (for example, institutes in central Europe are more easily accessible by train); significantly larger emissions also tend to be generated by senior astronomers ${ }^{14}$. The total footprint per astronomer from work-related activities depends on all these factors and ranges between about 5 and 40 metric tonnes of $\mathrm{CO}_{2}$ equivalents per year ${ }^{14-16}$. This can be compared to the European Union's 'Fit for 55' pledge, which implies emissions of about 3 tonnes per person per year for both work and private emissions until 2030.

Now that we have first estimates for the extent and the breakdown of our carbon footprint, we are empowered to eliminate $\mathrm{it}^{17,18}$. How quickly must astronomers act, and where should we begin? These questions were at the centre of a special session at this year's European Astronomical Society annual meeting, held virtually at the end of June. The challenge is to find a reduction path that is both significant and in agreement with the goals described by the Paris Agreement, while being fair both on a global level (richer countries need to decarbonize faster) as well as on a community level, where early-career researchers may have different communication needs than more senior colleagues who have already built their networks. Ideally, not only emissions for a certain activity are taken into account, but the full life-cycle of the project or instrument must be considered and emission budgets must be defined and reduced, such as has recently been done for the planned facilities Giant Radio Array for Neutrino Detection (GRAND) ${ }^{10}$, the Atacama Large Aperture Submillimeter Telescope (AtLAST), as well as the Square Kilometre Array Observatory (SKAO) ${ }^{11}$.
The absolute minimum emission reduction that needs to be achieved in Europe is a $55 \%$ reduction by 2030 (with respect to 1990), in agreement with the recent goal in the context of a European Green Deal. This goal, however, is a political compromise and not compatible with the emission reductions required per the Paris Agreement, which would foresee emission reductions on the order of at least $65 \%$.

In some areas, the path to zero emissions is clear: electrical energy must no longer be produced by burning fossil fuels, but through renewable, preferably local, electricity generation. Gains from the use of more efficient computing languages ${ }^{19}$ will unfortunately not help as Jevons paradox applies: efficiency gains usually lead to increased consumption; an approach of ecological sufficiency is required to counteract this trend. Sustainably produced electrical energy must power not only all current electricity consumption but will also be the driver for the electrification of heating through heat pumps and part of our future mobility needs. Several institutes and universities have started to invest in their own solar power arrays, although this can be tricky for historic monuments ${ }^{20}$. Remote observatories, usually at the sunniest places on this planet, can move to green energy as ESO has demonstrated for their La Silla site $^{21}$. For their ELT and VLT sites, ESO is in the process of commissioning a large solar array in the Atacama Desert ${ }^{13}$. Often the bulk of the electricity usage is during daytime hours when large air-conditioning facilities are required to keep the domes at night-time temperature ${ }^{22}$. Combined with a connection to the national grid - which can be fed with water and wind power - green energy can become available around the clock.

One of the most challenging areas to decarbonize is long-distance flights (25-50\% of the emissions of typical astronomy institutes ${ }^{15}$ ) as there is simply no alternative to burning fossil fuels: battery-driven aeroplanes are too heavy for more than short-haul transport, whereas synthetic fuels (or hydrogen) are too expensive and inefficient to produce and will not be available in the large quantities required to sustain a frequent-flyer attitude. Currently the only feasible option is to massively reduce our air travel. For national or regional trips, train travel is the obvious alternative, at least in regions with good train networks such as in Europe and parts of Asia. The majority of emissions for conferences and visits to observing sites stems from intercontinental flights ${ }^{14-16}$, however, and these flights cannot easily be replaced by any other means of physical transport. Despite this, the last 18 months 


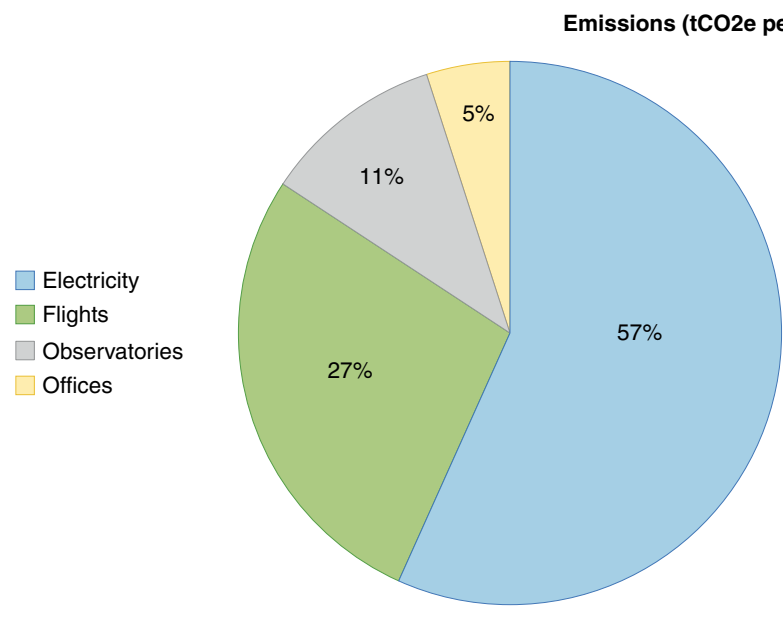

Australia/ICRAR: total $41.8 \mathrm{tCO}_{2}$ e per researcher (using the same emission model as MPIA)

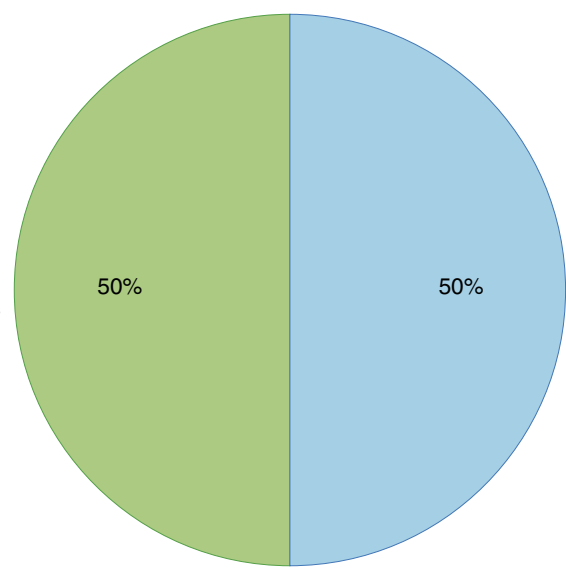

EWASS 2019 conference: $1.5 \mathrm{tCO}_{2}$ e per participant

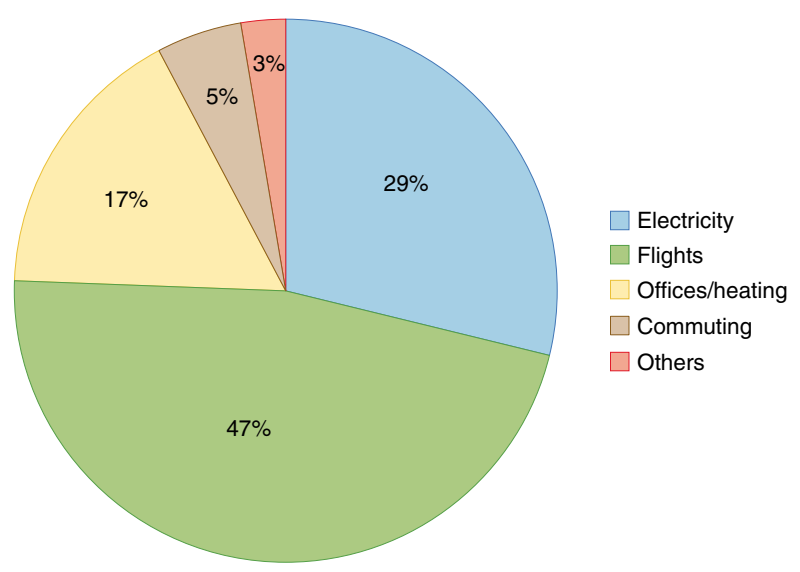

MPIA total: $18.1 \mathrm{tCO}_{2}$ e per researcher

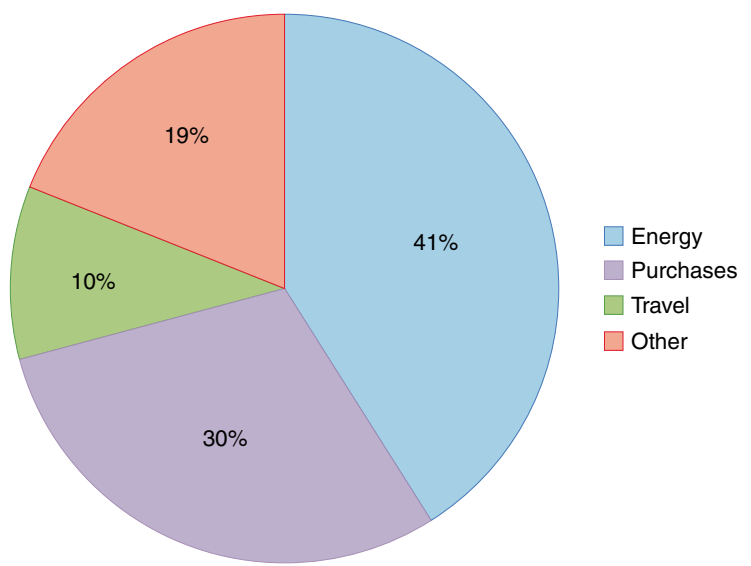

ESO (Annual Report 2020): $27.9 \mathrm{ktCO}_{2} \mathrm{e}$ (700 staff)

Fig. 3 | Carbon budgets for astronomical research activity. The carbon footprint of astronomical research activity as determined for the International Centre for Radio Astronomy Research (ICRAR) in Perth, Australia ${ }^{14}$ (top left), for the Max Planck Institute for Astronomy (MPIA) in Heidelberg, Germany ${ }^{15}$ (top right), for the annual meeting of the European Astronomical Society (EAS) 2019 in Lyon, France ${ }^{5}$ (bottom left), and for the European Southern Observatory (ESO) ${ }^{13}$ (bottom right).

have shown that our discipline can adapt to a complete cessation of flights. We do not actually need to be present physically to effectively communicate with each other. In fact, there is an increasing consensus that replacing real-life conferences by virtual meetings is not just better for the environment, but that well-designed online conferences can have benefits that reach far beyond the carbon footprint: they can be more inclusive, accessible, fairer, safer, and have higher legacy value too ${ }^{23,24}$. Yet, for many people, meeting others in person is a joy and traveling to other places in the world is a perk, and a positive aspect of their job as an astronomer. While online conferences are technically ready ${ }^{24}$, we must continue to develop immersive online conference experiences. Societally, however, we may need a transition period to normalize a culture shift from 'face-to-face, unless not possible' to 'online, unless face-to-face is a necessity'. While such a culture change would typically take several years, A4E members seek to accelerate this movement since there is no time to waste.

In addition to reducing our own emissions, astronomers can and should take part in climate communication to highlight the severity and urgency of the climate crisis to the wider public ${ }^{6}$, politicians and other decision makers ${ }^{25}$. We should also help define ambitious education programmes, applicable from primary schools to universities, to prepare the next generations for the systemic adaptations required to fight the climate crisis. Thanks to the wide interest from the public in astronomical research, we have a reach that extends beyond that of many other scientific areas; for example, the Event Horizon Telescope image of the supermassive black hole in
M87 made headlines in newspapers around the world. We have an obligation to use this reach in order to inform and warn the wider public of the damage done to our only home by continuing to burn fossil fuels. This becomes increasingly important given the generously funded lobbying activities of the fossil fuel industry, which have successfully counteracted scientifically informed policies that could stop global heating. Precisely because we are not climate scientists, we can and must use our neutral standpoint and our trusted public voice to speak out in support for climate science and to call for action ${ }^{6}$.

As we are yearning for more social interaction, we must avoid the calls to 'go back to normal', but use the change forced upon us due to the COVID-19 pandemic to define a 'new, better normal' that is ecologically sustainable, inclusive and fair. There is wide-spread agreement that 


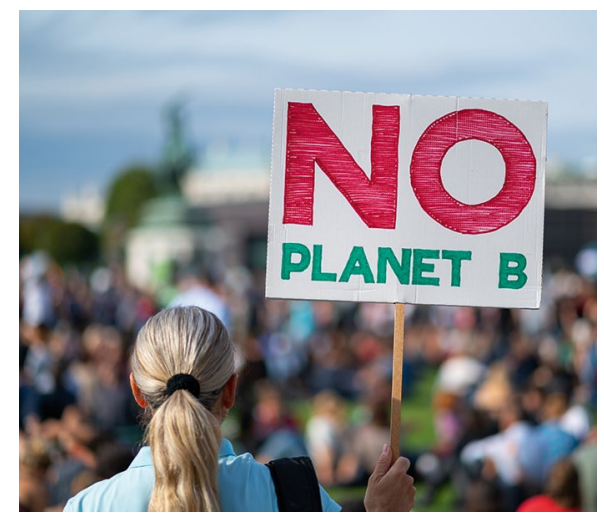

Fig. 4 | There is no planet B. Astronomy is the discipline that understands most keenly that there is no alternative to planet Earth for the survival of our species, and our actions must be consistent with this truth. Credit: amriphoto/E+/Getty

change must be coming both in the general population ${ }^{4}$ and also among astronomers ${ }^{26}$, but individuals still find it hard to reduce work-related flights due to the perceived damage upon their careers. The number of flights that one takes does not necessarily correlate with academic success, however. While some studies suggest that there is a correlation between the number of flights and the h-index ${ }^{26}$, others find that the underlying correlation is mainly one with salary ${ }^{27}$. In order to be equitable to researchers at all career levels and from all countries, it is critical to collectively agree on a new path at all levels: institutional, national and international. We must embrace virtual tools to enhance scientific collaboration, advocate for a just transition to renewable energy sources, and refuse a return to our wasteful practices of the past. We are the discipline that understands most keenly that there is no planet $\mathrm{B}$ (Fig. 4): our actions must be consistent with this truth.

\section{Leonard Burtscher (D) 1 ,}

Hannah Dalgleish (D2,3, Didier Barret (D)4, Tobias Beuchert ${ }^{5}$, Abhijeet Borkar (iD), Faustine Cantalloube [D $^{7}$, Abigail Frost (D) Victoria Grinberg (D),

Natasha Hurley-Walker ${ }^{10}$,

Violette Impellizzeri', Mathieu Isidro"1, Knud Jahnke (D) 12 and Michelle Willebrands ${ }^{1}$ ${ }^{1}$ Leiden Observatory, Leiden, The Netherlands. ${ }^{2}$ Department of Physics, University of Oxford, Oxford, UK. ${ }^{3}$ Department of Physics, University of Namibia, Windhoek, Namibia. ${ }^{4}$ Institut de Recherche en Astrophysique et Planétologie, Centre National de la Recherche Scientifique, Université de Toulouse, Toulouse, France. ${ }^{5}$ European Southern Observatory, Garching bei München, Germany. ${ }^{6}$ Astronomy Institute of the Czech Academy of Sciences, Prague, Czech Republic. ${ }^{7}$ Laboratoire d'Astrophysique de Marseille, Marseille, France. ${ }^{8} \mathrm{KU}$ Leuven, Leuven, Belgium. ${ }^{9}$ European Space Agency - ESTEC, Noordwijk, The Netherlands. ${ }^{10}$ International Centre for Radio Astronomy Research, Curtin University, Bentley, Western Australia, Australia. ${ }^{11}$ SKA Observatory, Macclesfield, UK. ${ }^{12}$ Max Planck Institute for Astronomy, Heidelberg, Germany.

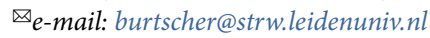

Published online: 15 September 2021 https://doi.org/10.1038/s41550-021-01486-x

\section{References}

1. IPCC: Summary for Policymakers. In: Climate Change 2021: The Physical Science Basis. Contribution of Working Group I to the Sixth Assessment Report of the Intergovernmental Panel on Climate Change (eds Masson-Delmotte, V. et al.) (Cambridge University Press, in the press).
2. Philip, S. et al. Hydrol. Earth Syst. Sci. 23, 1409-1429 (2019).

3. van Oldenborgh, G. J. et al. Nat. Hazards Earth Syst. Sci. 21 941-960 (2021).

4. Leiserowitz, A. et al. International Public Opinion on Climate Change (Yale Program on Climate Change Communication and Facebook Data for Good, 2021).

5. Burtscher, L. et al. Nat. Astron. 4, 823-825 (2020).

6. Anderson, A. \& Maffey, G. Nat. Astron. https://doi.org/10.1038/ s41550-021-01481-2 (2021).

7. Frost, A. J. et al. Commun. Astron. Public (in the press).

8. Burtscher, L. et al. Commun. Astron. Public (in the press).

9. Cantalloube, F. et al. Nat. Astron. 4, 826-829 (2020).

10. Aujoux, C. et al. Astropart. Phys. 131, 102587 (2021).

11. Isidro, M. Contact 8, 27-31 (2021).

12. Flagey, N. et al. Nat. Astron. 4, 816-818 (2020).

13. Annual Report 2020125 (European Southern Observatory, 2021).

14. Stevens, A. R. H. et al. Nat. Astron. 4, 843-851 (2020).

15. Jahnke, K. et al. Nat. Astron. 4, 812-815 (2020).

16. van der Tak, F. et al. The Carbon Footprint of NL Astronomy in 2019 (Zenodo, 2021); https://doi.org/10.5281/zenodo.5084398

17. Rybizki, J. et al. Climate Impact of Flying and Computing Aspects to be Tackled by the Astro Community (Zenodo, 2021); https://doi.org/10.5281/zenodo.5056536

18. Massey, R. 200 Years in and Looking to the Future: Decarbonising the Oldest Astronomical Society in the World (Zenodo, 2021); https://doi.org/10.5281/zenodo.5141964

19. Portegies Zwart, S. Nat. Astron. 4, 819-822 (2020).

20. Steinmetz, M. Your Local Carbon Footprint - What Can be Done at Your Institution? (Zenodo, 2021); https://doi.org/10.5281/ zenodo. 5060073

21. Filippi, G. et al. Proc. SPIE 9906, 990659 (2016).

22. Weilenmann, U. Messenger 148, 39-41 (2012).

23. White, S. An Environment for Everyone (Zenodo, 2021); https://doi.org/10.5281/zenodo.5083144

24. Moss, V. A. et al. Nat. Astron. 5, 213-216 (2021).

25. Williams, A. Nat. Astron. https://doi.org/10.1038/s41550021-01490-1 (2021).

26. Berné, O. \& Ben Ari, T. Labos 1point5: A Collective Action to Reduce the Carbon Footprint of Research at the Scale of France (Zenodo, 2021); https://doi.org/10.5281/zenodo.5067162 27. Wynes, S. et al. J. Clean. Prod. 226, 959-967 (2019).

28. Capstick, S. et al. in Emissions Gap Report 2020 Ch. 6 (UNEP, 2020).

Acknowledgements

The authors would like to acknowledge collaboration with the A4E Collective.

Competing interests

The authors declare no competing interests. 